\title{
Validation of functionability of transition metal and its alloys at the simultaneous introduction of two oxidizing agents in the volume of liquid phase
}

\author{
Swetlana Pozhidaeva ${ }^{1 *}$ and Galina Buryih ${ }^{1}$ \\ ${ }^{1}$ Southwest State University, 305040 Kursk, Russia
}

\begin{abstract}
At the chemical process in the volume of a liquid reaction mixture in the presence of two specific groups of oxidizers in a metal reactor or reactor using metal elements, favourable conditions for abnormally rapid oxidation of the metal with subsequent destruction at room or close to them temperatures may appear. The condition for destruction is the process according to the cycle scheme with the regeneration of the metal oxidizer, which may initially be present in the system in catalytic or close to them quantities. In the presence of several metal elements, the destruction occurs all at the same time, but in different proportions. As a result, this leads to a deterioration in the characteristics of the process, and the process of destruction in the apparatus (reactor) can be accompanied by the emergence of a far unsafe, unpredictable situation.
\end{abstract}

The presence of two or more oxidizers in a system with a metal or alloy is quite common. For example, for successful etching of printed circuit boards [1-3], such mixtures are used for high-speed removal of a metal layer from the surface without any physical actions by immersing in the appropriate solution for a short time for several minutes. The presence of the second oxidizer in the liquid bulk phase is the reason for a sharp increase in the corrosion rate, leading to uncontrolled metal consumption [4 - 6]. But, if in the first case etching is carried out purposefully using concentrated solutions and excess reagents, then uncontrolled destruction of metals in the corrosion process occurs at lower concentrations of reagents and their stoichiometric deficiencies. Using the experiment, a group of processes was identified, accompanied by accelerated destruction of metal at speeds close to the etching rates under conditions close to corrosive due to the participation of two oxidizers in the macrocycle (fig.1).

The scheme works successfully if the metal oxidizer $\left(\mathrm{OK}_{\mathrm{Me}}\right)$ is halogen, in particular, iodine (bromine), or copper compounds (II), including corrosion products of copper products [7 - 9]. The presence of these compounds is not a single case, moreover, halogens in the system can also appear in the case of the process from halides used as additives [1013] according to the scheme

\footnotetext{
*Corresponding author: pozhidaeva_kursk@mail.ru
} 


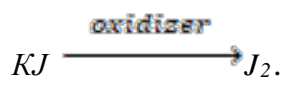

However, in these cases, the processes develop with rather long induction periods.

$$
\begin{aligned}
& \mathrm{Me}+\mathrm{OK}_{\mathrm{Me}} \stackrel{+H A}{\longrightarrow} \mathrm{MeX} \frac{+O K_{n} ;}{+H A} \mathrm{MeA}_{\mathrm{m}}+\mathrm{OK}_{\mathrm{Me}} \\
& \left(\mathrm{OK}_{\mathrm{Me}} ; \mathrm{OK}_{\mathrm{Me}}\right)
\end{aligned}
$$

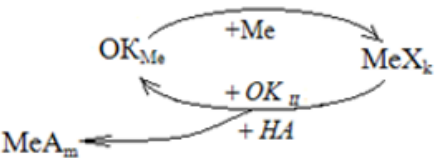

Fig. 1. The scheme of the processes studied in the work, accompanied by the destruction of metals and alloys

The metal oxidizer $\left(\mathrm{OK}_{\mathrm{Me}}\right)$ is required in a large stoichiometric disadvantage with respect to the reacted metal in an amount approximate to the catalytic one. The second oxidizer $\left(\mathrm{OK}_{\mathrm{II}}\right)$ provides regeneration of the first oxidizer. Such compounds as metal oxides and salts in a higher degree of oxidation, metal and hydrogen peroxides, and molecular oxygen were used and studied as the second oxidizer at this time. The described phenomena of unexpected and almost unpredictable strong destruction of metals have been repeatedly observed in practice. The dynamics of the destruction of the reactor elements according to the scheme is shown at the Figures 2-4.
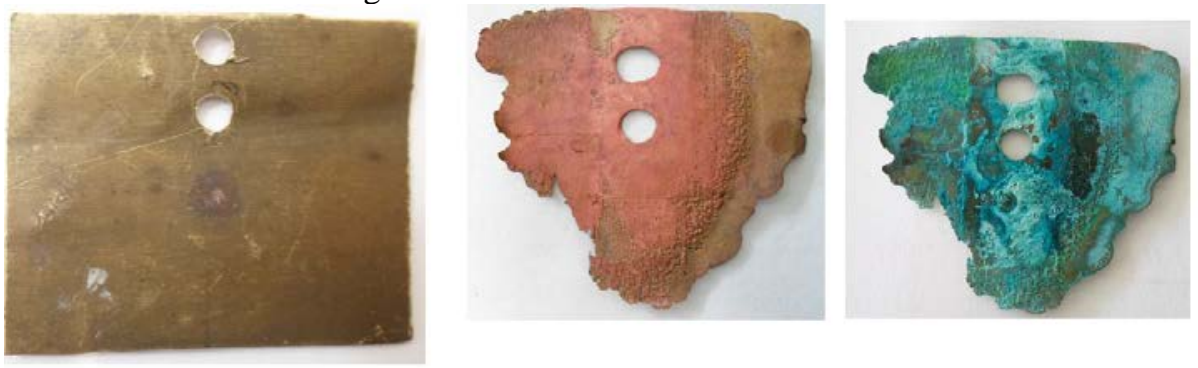

Fig. 2. Dynamics of destruction of the paddle of a bead mill made of bronze (the rate of destruction of bronze is $2.97 \mathrm{~g} / \mathrm{h}$ )

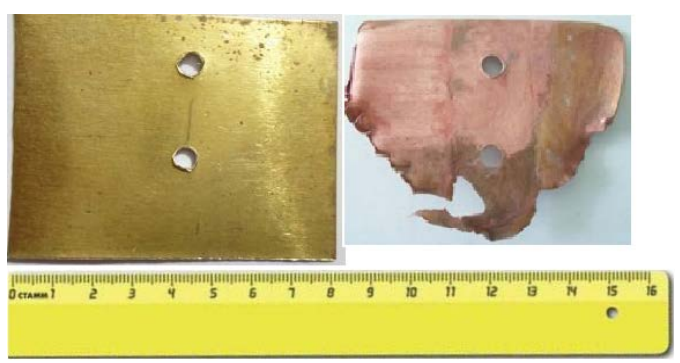

$a$
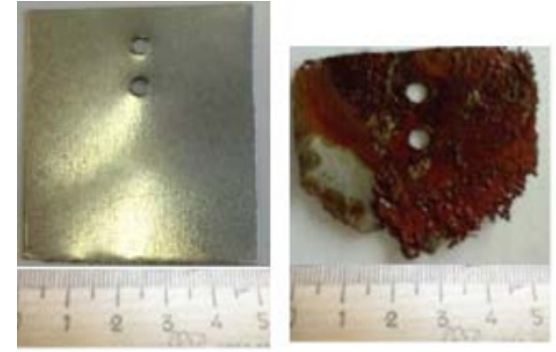

$b$

Fig. 3. An example of the destruction of the paddle of a bead mill from brass (the rate of destruction $3.22 \mathrm{~g} / \mathrm{h})(a)$ and galvanized sheets $(b)$ (destruction rate of $1.86 \mathrm{~g} / \mathrm{h}$ ) 

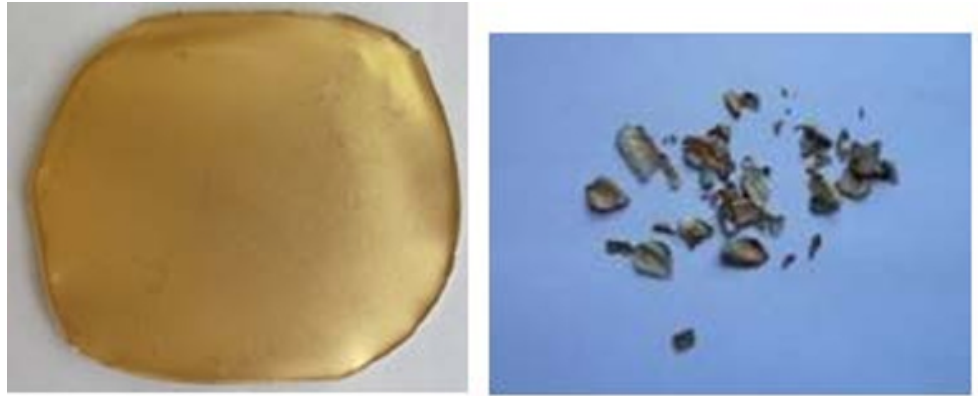

Fig. 4. An example of the destruction of the false bottom of reactor from brass (the rate of destruction $3.22 \mathrm{~g} / \mathrm{h}$ ) ( $a$ ) and galvanized sheets $(b)$ (destruction rate of $0.57 \mathrm{~g} / \mathrm{h}$ )

As a result, partial damage of the equipment or its complete failure was observed quite often. In general, it was found that the degree of destruction of the metal (part) according to the scheme during the contact with the liquid volume phase containing an acidic reagent and a metal oxidizer in a stoichiometric disadvantage with respect to the reacted metal, from 1 to $100 \%$ was achieved in a time from 3 to 30 hours, and regardless of the strength of the acid used and the degree of pre-grinding of the metal (table 1). And these are not specially selected and suboptimal conditions, in which the destruction rates of the metal (alloy) will be much higher.

Table 1. The information about the destruction of metals(alloys)

\begin{tabular}{|c|c|c|c|c|}
\hline \multirow{2}{*}{$\begin{array}{l}\text { The nature of } \\
\text { metals(alloys) }\end{array}$} & \multicolumn{2}{|c|}{ The configuration of metals(alloys) } & \multirow{2}{*}{$\begin{array}{l}\text { The degree } \\
\text { of destruc- } \\
\text { tion of the } \\
\text { metal and } \\
\text { alloy, \% }\end{array}$} & \multirow{2}{*}{$\begin{array}{l}\text { Maximum } \\
\text { velocity } \\
\text { metal } \\
\text { consumption, } \\
\text { g/kg Me hour }\end{array}$} \\
\hline & $\begin{array}{l}\text { Fragment } \\
\text { (lump of metal) }\end{array}$ & $\begin{array}{l}\text { in the shape of a } \\
\text { the paddle of the } \\
\text { bead mill }\end{array}$ & & \\
\hline \multirow{2}{*}{ Cooper } & + & & $10-100$ & \multirow[t]{2}{*}{$400 \div 900$} \\
\hline & & + & $5-60$ & \\
\hline \multirow{2}{*}{ Bronze } & + & & $5-40$ & 320 \\
\hline & & + & $45-64$ & 800 \\
\hline Brass & & + & $40-50$ & 600 \\
\hline Devardo's alloy & + & & $50-100$ & \\
\hline Arndt Alloy & + & & $80-100$ & \\
\hline \multirow{2}{*}{$\mathrm{Fe}$} & + & & $25-35$ & 330 \\
\hline & & + & $28-46$ & \\
\hline Cast iron & + & & $15-36$ & \\
\hline Steel & & & $10-63$ & \\
\hline Galvanized sheets & & & $20-50$ & \\
\hline Zinc & + & & $1-40$ & 330 \\
\hline
\end{tabular}




\begin{tabular}{|l|c|l|l|l|}
\hline \multirow{2}{*}{ Aluminum } & & + & $8-54$ & \multirow{2}{*}{600} \\
\cline { 2 - 4 } & + & + & $0,7-89$ & \multirow{2}{*}{$1-50$} \\
\hline \multirow{2}{*}{ Dural } & + & & $15-68$ & \\
\hline \multirow{2}{*}{ Lead } & + & + & $15-48$ & \multirow{2}{*}{350} \\
\cline { 2 - 4 } Nichrome & + & & $24-56$ & \\
\hline FeCrAl alloy & + & & $10-15$ & \\
\hline Tin metal & + & & $1-75$ & 240 \\
\hline
\end{tabular}

Under model conditions, the processes in question are performed with copper and its alloys (brass, bronze, Arndt alloy (60\% Cu, 40\% Mg), Devard alloy (50\% Cu, 45\% Al, 5\% Zn), etc.), reduced iron, cast iron and steel, aluminum and its alloys, zinc and galvanized iron, nickel-plated products, Wood alloy, tin, lead, etc. Their conditions are very wide. More than 150 items (mineral and carboxylic acids, low-molecular fatty alcohols, phenols, acid salts) were studied by the nature of the acid reagent, more than 100 items, etc., were added.

The destruction of the paddle of a bead mill for a short period of time, commensurate with the time of the technological process, leads to a deterioration of characteristics of process (Fig. 5) on the one hand. And on the other hand, the process of destruction of the part in the apparatus (reactor) can be accompanied by the occurrence of a far unsafe emergency situation, and often poorly predictable.

\section{The degree of}

\section{destruction of the $m$ etal and alloy, $\%$}

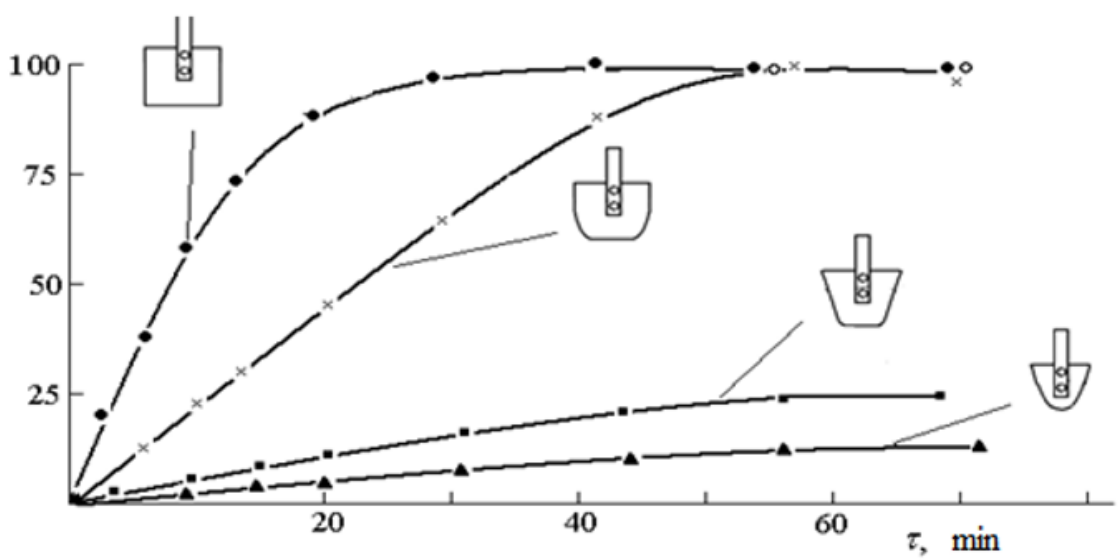

Fig. 5. An Example of reduction of mixing efficiency and metal conversion due to the destruction of the paddle of the bead mill

At the simultaneous presence of several elements made of metal, the destruction occurs of all elements, regardless of the degree of fragmentation of metal (alloys) and contact with the volume phase (Figure 6). 


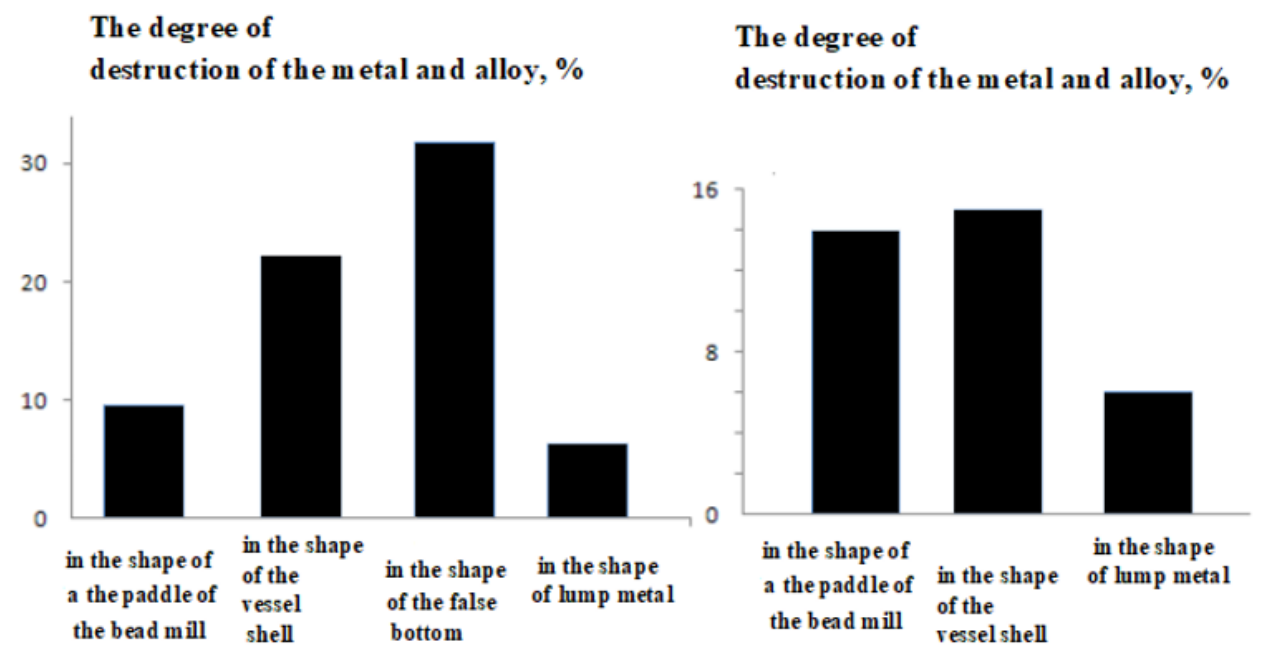

Fig. 6. An Example of the influence of the surface configuration and the location of the brass alloy in the process zone on the degree of destruction of brass with the simultaneous presence of different elements in the system

The differences in the degree and places of destruction of the paddle of the bead mill are explained by the dependencies its from the initial thickness, mass and configuration. Thin and elastic paddle of the bead mill at the beginning are more destroyed at the edges. Reducing the size leads to a loss of mixing efficiency and intensity, which affects the rates of further destruction. Thick and inelastic paddle of the bead mill, losing weight, lose little in size.

Thus, the simultaneous presence of these components in the volume of the reaction mixture can lead to uncontrolled destruction of metal, including metals and alloys which used for the manufacture of equipment and reactor elements. Creating favorable conditions for the operation of the cycle can lead to a rapid loss of equipment operability and its failure, as well as a decrease in the characteristics of the process

\section{References}

1. B. Snopok, T. Snopok, A. Laroussi, V.M. Mirsky, K. Voïtchovsky, C. Cafolla, Surfaces and Interfaces. 22, 100818 (2021).

2. G.H.A. Abrenica, S. De Gendt, S. Arnauts, F. Holsteyns, D.H. Van Dorp, M. Fingerle, T. Mayer, M.V. Lebedev, ECS Journal of Solid State Science and Technology. 9(8), 084002 (2020)

3. C.A. Escobar Claros, A. Moreira Jorge, J.-C. Leprêtre, V. Roche, L. Contri Campanelli, C. Bolfarini, Corrosion Science. 188. 109544 (2021).

4. B.G. Koushik, N. Van den Steen, M.H. Mamme, Y. Van Ingelgem, H. Terryn, Journal of Materials Science and Technology 62, 254-267 (2021).

5. B. Quan, J. Li, C. Chen, Metals. 11(5), 753 (2021).

6. X. He, T. Ahn, J.P. Gwo, Corrosion. 74(2), 158-168 (2018).

7. S. D. Pozhidaeva, A. Yu. Eliseeva, A. M. Ivanov, RUSSIAN METALLURGY (METALLY). 13, 1117-1123 (2015). 
8. S. D. Pozhidaeva, A. M. Ivanov, RUSSIAN METALLURGY (METALLY). 13, 15101517 (2019).

9. S. D. Pozhidaeva, A. M. Ivanov, D. A. Sotnikova, A. Yu. Eliseeva, Russian Journal of inorganic chemistry. 58(12), 1428 - 1433. (2013)

10. T. Warchilová, V. Dillingerová, T. Vaculovič, V. Kanický, R. Škoda, T. Šimo, O. Matal, Spectrochimica Acta Part B: Atomic Spectroscopy. 148, 113-117 (2018)

11. G.-N. Liu, R.-Y. Zhao, R.-D. Xu, Q. Liu, B. Xu, Y.-Y. Wang, Q. Wu, J.-N. Wang, Y. Nie, C. Li, Journal of Materials Chemistry C. 7(25), 7700 - 7707 (2019)

12. H. Shimizu, A. Saito, A. Yoshimura, V.V. Zhdankin, K. Noguchi, V.N. Nemykin, Beilstein Journal of Organic Chemistry. 14, 531-536 (2018)

13. E.T. Denisov, T.G. Denisova, Kinetics and Catalysis. 59(1) (2018) 